\title{
CENTENARIO DE LA CONSTITUCIÓN DE LOS ESTADOS UNIDOS MEXICANOS
}

\section{César Aguado Renedo*}

La intervención del anfitrión de este seminario ${ }^{1}$ sobre el objeto del mismo, el doctor Corzo Sosa, ha expuesto el dilema consistente en que si lo que procede llegado el centenario de la Constitución mexicana de $1917,{ }^{2}$ es una reforma parcial que la misma parece demandar, o una nueva Constitución. Según él mismo indica, en estos momentos la mayoría de la doctrina del país se inclina por la primera posibilidad frente a la segunda. Él mismo — según revela - era también partidario inicialmente de la reforma parcial, pero una reflexión más detenida sobre el tema le ha llevado finalmente a entender que lo adecuado sería, más bien, generar un texto nuevo, en el que, sobre todo, se dispusiera un procedimiento de reforma severamente agravado, incluyendo, en su caso, como parte de esa reforma la aprobación popular de la misma mediante referéndum o la intervención de una Asamblea Constituyente, tal y como sucede en otros sistemas que han redactado nuevas Constituciones o que las han modificado contemplando tales intervenciones.

Sobre la cuestión expongo algunas reflexiones por si pudieran tener algún interés, que en todo caso sería más bien modesto, caso de que se hiciera real la posibilidad de alterar o de sustituir por otra la Constitución mexicana vigente. A mi muy modesto juicio, el dilema entre la reforma total o parcial del texto constitucional es bastante relativo, como luego se dirá, pero para seguir

\footnotetext{
* Profesor de Derecho constitucional, Universidad Autónoma de Madrid.

1 El seminario "Constitución y aspectos constitucionales" se llevó a cabo los días 22 y 23 de enero de 2015 en el Instituto de Investigaciones Jurídicas de la UNAM.

2 Corzo Sosa, Edgar, "Prólogo", en Pinto Muñoz, Jacinto Héctor, Una nueva Constitución aprobada por el pueblo soberano, UNAM, Instituto de Investigaciones Jurídicas, 2016, pp. XIII-XX.
} 
Esta revista forma parte del acervo de la Biblioteca Jurídica Virtual del Instituto de Investigaciones Jurídicas de la UNAM

con la propuesta, esa solución deriva de dos consideraciones, esencialmente: de la significación o simbolismo del texto para la comunidad política, y de las concretas circunstancias políticas en que se produzca el intento de reforma o de aprobar un nuevo texto.

En cuanto a lo primero, basta pensar en el paradigma de Constitución significativa, que obviamente es el texto constitucional norteamericano, cuyo comienzo ("We the people...") todo el mundo conoce allí, y cuyas reformas tienen lugar mediante enmiendas con el fin de que el texto originario permanezca inalterado. Por tanto, no hay pretensión seria de modificar tal texto, que sirve de lazo común, así sentido, entre los distintos habitantes del país, con independencia de su procedencia o lugar de nacimiento, de su edad o de su estatus social.

En idéntica línea se tiene el ejemplo de la Ley Fundamental de Bonn de 1949, llamada así, como es sabido, porque derrotada y ocupada Alemania tras el término de la Segunda Guerra Mundial, que dicho país inició y protagonizó, ni desde la perspectiva de los ocupantes podía permitirse el ejercicio de una capacidad de autoorganización que comportara darse a sí misma una Constitución propiamente tal (de hecho, el texto se generó a partir del Documento de Frankfurt que proporcionaron las potencias ocupantes y, elaborado el texto final, hubo de ser ratificado por sus respectivos gobernadores militares antes de pasar a la ratificación por los parlamentos de los Länder), ni desde de la perspectiva de los propios alemanes quiso denominarse formalmente "Constitución" (Verfassung), sino Grundgesetz ("Ley Fundamental") für die Bundesrepublik Deutschland, para significar igualmente que no era la Constitución de toda Alemania, toda vez que no iba a entrar en vigor en una extensa parte del territorio (el ocupado por la entonces URSS), y una Constitución que verdaderamente pudiera considerarse "alemana" sólo podía tener lugar cuando el país se reunificara. Esta reunificación tiene lugar, como es bien sabido, tras la caída del Muro de Berlín, en 1989, lo que llevó casi de inmediato a que se reclamara por un sector importante de políticos y juristas que Alemania se dotara de una Constitución formalmente así designada y aprobada por todos los Länder, ya integrantes del Estado alemán, y con ratificación en referéndum. La significación lograda a lo largo de cuatro décadas por la Ley Fundamental, sin embargo, hizo que tal pretensión quedara en una "mera" reforma constitucional en 1994, y que se conservara la denominación, aunque en la práctica se la designe indistintamente con esa denominación o con la de "Constitución alemana". 
Esta revista forma parte del acervo de la Biblioteca Jurídica Virtual del Instituto de Investigaciones Jurídicas de la UNAM

Salvadas las inmensas distancias con estos dos ejemplos, con la Constitución española de 1978 se da un fenómeno al menos parcial y relativamente parecido en cuanto a la significación: al resultar, como fue, un texto, producto del más amplio consenso ${ }^{3}$ por primera vez en su historia, con motivo de las distintas crisis que en la actualidad inciden en el país y que demandan, a juicio de la gran mayoría, importantes reformas de la Constitución, lo cierto es que hay una notable resistencia a su modificación, además de por evidentes razones políticas en unos casos y de lo dificultoso del procedimiento agravado previsto para ello, sin duda, también porque se le atribuye ese plus del que le dota su gran aprobación inicial, solicitándose otro consenso cuando menos similar para que la reforma tenga lugar; esto es, para que pueda "competir en legitimidad", por así decir, con el texto que se quiere sustituir; ardua dificultad porque, al menos hoy por hoy, no parece posible que se dé un acuerdo no ya igual, sino siquiera parecido al originario. Más aún: hasta donde se me alcanza no hay propuestas de un texto constitucional español nuevo, en razón — se sobrentiende - de que, aun más o menos reformado, se quiere preservar la significación del originario en virtud de ese plus aludido.

El quantum de significación que para la población mexicana tiene su texto constitucional, será cuestión a apreciar por sus iuspublicistas (cuya parte más significativa seguramente se encuentra en este reconocido Instituto en el que tiene lugar el encuentro que hoy nos reúne) y, sobre todo, desde el punto de vista práctico, por su clase política, que será la que haya de gestionar de facto, en su caso, su reforma o innovación. En principio, el carácter centenario de la Constitución, el que la misma insertara por primera vez de algún instituto de tanta relevancia como el amparo, e incluso su identificación con un nombre propio (la Constitución de Querétaro) por el que es notoriamente conocida no sólo dentro, sino también fuera del país, son datos que contribuyen a entender ciertamente significativa o simbólica la actual norma fundamental mexicana. En contra de esa impresión, sin embargo, juegan, y desde luego de forma importante, las numerosas reformas que ha venido experimentando a lo largo de tan dilatado periodo, que han hecho del texto constitucional — como se ha encargado de subrayar el doctor Corzo - un texto muy, si no totalmente, diferente al original.

\footnotetext{
3 Aunque el diccionario de la Real Academia Española define el consenso como el acuerdo unánime entre los componentes de un grupo, o entre grupos, va de suyo que políticamente la inmensa mayoría de las veces es imposible la unanimidad, de modo que, en la práctica, cuando se usa tal término quiere hacerse referencia a un acuerdo que sea tan mayoritario que resulte irrelevante, o escasamente relevante, el disenso que se muestre respecto del objeto de que se trate.
} 
Esta revista forma parte del acervo de la Biblioteca Jurídica Virtual del Instituto de Investigaciones Jurídicas de la UNAM

Por sí solas, las numerosas reformas no disminuyen el valor significativo del texto: la Ley Fundamental de Bonn alemana ha sido también muy reformada (al día de hoy, en sesenta ocasiones desde que se promulgara en 1949), y en algunos casos de forma muy relevante (la última, en materia de organización territorial, de bastante más trascendencia práctica que la que tuvo lugar en 1994, tras la reunificación alemana). Pero contra lo que en su ponencia subraya el doctor Edgar Corzo que ha sucedido con la Constitución mexicana, a saber, que ha incurrido en contradicciones claras con las incoherencias internas que ello conlleva, en el caso alemán han sido reformas coherentes, producto de análisis jurídicos, previos, extensos y detallados por expertos, que han llevado su buen tiempo y que respondían a objetivos claros, producto de las necesidades sentidas como tales por los operadores jurídicos y políticos. No es, pues, cuestión tanto de la mayor o menor fidelidad al texto original del texto resultante al cabo de un siglo (salvo que sea ese el presupuesto del que se parta, como sucede en el caso de la Constitución de Estados Unidos), cuanto de que el resultado de las modificaciones siga siendo un texto suficientemente aceptable que mantiene un grado suficiente de adhesión de la comunidad que por ella se rige.

Por lo demás, en fin, no puede perderse de vista, que salvo en los procesos puramente revolucionarios que dan lugar a un sistema radicalmente distinto al anterior con el cual se quiere acabar, esto es, dan lugar a un ruptura deliberada con el pasado inmediato (típicamente, los que en su día dieron lugar a sistemas del llamado "socialismo real"..., para, acabado éste en los países europeos del Este, proceder de igual manera a sustituir aquél con -entre otras cosas - nuevos textos constitucionales radicalmente distintos; más recientemente en América Latina lo mismo puede decirse de los movimientos bolivarianos, con las Constituciones de Venezuela de 1999, de Ecuador de 2008 o de Bolivia de 2009), lo habitual es que un texto constitucional, por nuevo que se quiera, recoja buena parte del anterior, y más en particular lo que se entiende que ha sido bueno, útil, lo que ha servido al fin pretendido con los contenidos correspondientes. De suerte que podrá calificarse a la Constitución de nueva, aun cuando no poco de su articulado se corresponda, con palabras más o menos similares, a la anterior. Quiere decirse, en definitiva, y como adelantamos ya, que la diferencia entre "reforma" y "novedad" seguramente obedece menos a la semántica en el campo de las transformaciones de los textos constitucionales que en otros campos.

Por último, no puedo dejar de apuntar, que sea cual sea la determinación por la que finalmente se opte (texto constitucional "nuevo", o sólo "refor- 
Esta revista forma parte del acervo de la Biblioteca Jurídica Virtual del Instituto de Investigaciones Jurídicas de la UNAM

ma"), habrá de darse lugar a un proceso protagonizado por el constituyente (en el primer caso) o constituido-constituyente (en el segundo). En principio, la diferencia entre uno y otro es importante: es la que va de escribir en un papel en blanco a corregir (enmendando, añadiendo, quitando, sustituyendo) un papel ya escrito por otros con anterioridad. Pero en uno y otro caso, la demanda principal es la misma: lo que vaya a constituir el nuevo contenido realmente fundamental ha de ser claro y terminante, ha de constituir opciones indudables que resulten guía para los actores políticos y para los intérpretes jurídicos (en particular, para la Suprema Corte). Se infiere fácilmente de ello que no pueden ser muchos los contenidos que hemos designado como "realmente fundamentales": los derechos, la forma de Estado y de gobierno (con la correspondiente relación entre poderes elegidos por los ciudadanos), la organización territorial, los elementos esenciales de la justicia y del sistema fiscal y económico y, por supuesto, su sistema de reforma, que, como el doctor Corzo ha enfatizado en su exposición oral, seguramente ha de ser más rígido de lo que hoy se dispone, en el bien entendido de que la rigidez no es jerarquía, sino una garantía de la misma, a la que ciertamente el derecho constitucional comparado muestra que se acude comúnmente (otra cosa es el grado de rigidez y la aplicación del mismo grado o de otro distinto, según la parte del texto constitucional de que se trate). Esos contenidos han de quedar fuera de la discusión política habitual, comenzando en primer lugar por la competencia política casi siempre feroz de los periodos electorales, pues de otro modo la estabilidad del sistema y con ella la perdurabilidad del mismo, que son fines esenciales de toda Constitución, se verán comprometidos $\mathrm{y}$, más temprano que tarde, desaparecerán. Nada hay inmutable ni eterno, mas las discusiones de "política constitucional" no pueden ser algo cotidiano, porque ello comportaría banalizar lo que se presupone fundamental.

De lo que se acaba de afirmar se deduce, o al menos así lo deduzco yo modestamente, que no cabe ni reforma ni menos aún nuevo texto fundamental si no hay acuerdo político mayoritario suficiente para determinar esos contenidos. Suficiente no quiere decir una mayoría simple: quiere decir un acuerdo de entidad importante entre los actores políticos. Pero, de igual modo, de entidad suficiente no quiere decir que una minoría, incluso relevante, tenga en sus manos la posibilidad de bloquear de forma sistemática y sin razones muy fundadas, un acuerdo que asuma una mayoría suficientemente importante. Un proceso de elaboración de una nueva Constitución o de una reforma importante de la misma no puede durar largo tiempo sin 
Esta revista forma parte del acervo de la Biblioteca Jurídica Virtual del Instituto de Investigaciones Jurídicas de la UNAM

correr el riesgo de degenerar y finalizar enmoheciéndose y descomponiéndose: si no se dispone del consenso ideal se abre la opción de posponer la reforma, o de recurrir a la transacción entre las diversas fuerzas políticas de un texto provisional difiriendo al legislador futuro la concreción de lo acordado. ${ }^{4}$ Es, sin duda, una fórmula riesgosa, pero es un riesgo inherente a esta forma de sortear el empantanamiento de la elaboración de una nueva carta constitucional o de una reforma de la misma.

Como es evidente, hay muchas más cosas que decir sobre una ponencia tan sugerente como la que nos ha trasladado el doctor Corzo y, con él, las extremadamente interesantes ponencias, expuestas con envidiable brillantez por los doctores Sierra Porto y Abad Yupanqui sobre las respectivas Constituciones actuales de sus países, Colombia y Perú, en particular, en lo referido a su peculiar génesis o mejor a los hechos de los que traen causa. Unidas estas experiencias a la citada "Constitución" alemana o a otros supuestos (como la sudafricana o las reformas constitucionales belgas), y a la imprescindible referencia de la no-Constitución (formal) británica, permiten deducir deducir algunas conclusiones, por así decir, desmitificadoras de esta categoría normativa sin la cual no pueden entenderse jurídico-políticamente los últimos dos siglos largos de nuestra civilización.

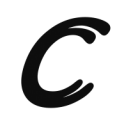

4 Puede servir como ejemplo de lo que se señala lo sucedido con el título VIII de la Constitución española de 1978, que regula la organización territorial del Estado y, en particular, las Comunidades Autónomas: como apunté hace años utilizando la terminología de C. Schmitt que ha hecho fortuna, ese título constitucional es un ejemplo de "compromiso apócrifo", esto es, de compromiso consistente en no resolver el asunto en el momento y diferirlo al legislador futuro para que sea éste el que, mediante la elaboración y aprobación de leyes especialmente exigentes en su elaboración y aprobación (en el caso español, los Estatutos de Autonomía), termine de concretar la cuestión problemática (el modelo territorial en el supuesto español). La solución, que evidentemente no es la ideal, es un recurso aceptable, porque logrado el consenso sobre el resto de las relevantes cuestiones problemáticas permite aprobar la reforma o el nuevo texto de que se trate (en el caso al que me refiero, permitió la aprobación de la Constitución Española de 1978 en un tiempo prudencial y en unas circunstancias difíciles — de terrorismo y agitación social sobre todo-; de no haberse recurrido a este expediente, es más que posible que a estas alturas los españoles no dispusiésemos aún de una Constitución y, con mucha probabilidad, de una que hubiera propiciado el más exitoso de los periodos de la historia moderna como ha propiciado la actual). La enseñanza manifiesta que se deduce de la experiencia española a la vista de su resultado es el extremo y, sobre todo, firme cuidado con el que ha de seguirse ese cumplimiento por el legislador futuro (vale decir, por las fuerzas políticas que accedan al poder y por las decisiones de los máximos órganos de control, tanto político como jurisdiccional) de lo diferido por el constituyente en virtud de esos compromisos apócrifos. 